Revista Matemática Universitária, vol. 2, 2021

ISSN: $2675-5254$ - DOI: $10.21711 / 26755254 / \mathrm{rmu} 20217$

\title{
UM INCANSÁVEL COMBATENTE NA DEFESA DA INVESTIGAÇÃO MATEMÁTICA: ANTÓNIO ANICETO MONTEIRO (1907-1980)
}

\author{
LUÍS MANUEL RIBEIRO SARAIVA
}

Uma perspectiva mais completa da acção de qualquer pessoa implica ter-se em conta o contexto em que viveu, as restrições que teve de enfrentar ou, pelo contrário, o ambiente favorável que encontrou. Neste sentido podemos dizer que o matemático António Aniceto Monteiro foi um verdadeiro gigante na ciência do século XX, e mais especificamente na matemática. Viveu a época conturbada do pós Primeira Guerra Mundial, com a ascensão do fascismo e do nazismo na Europa, que culminaram na Guerra Civil de Espanha (1936-1939) e na $2^{\text {a }}$ Guerra Mundial (1939-1945). No seu país suportou a época inicial de uma ditadura que se estendeu de 1926 até 1974, um regime retrógrado que colocava o povo sob intensa vigilância e repressão. Mesmo quando partiu para o Brasil para aí viver, Monteiro continuou a ser vigiado pela polícia política portuguesa.

Foi educado num sistema universitário que tinha como única finalidade a transmissão de conhecimento aos futuros quadros da administração pública e aos futuros professores. O currículo universitário no que diz respeito à matemática era antiquado, tinha imensas falhas, e a investigação matemática praticamente não existia enquanto actividade organizada.

No primeiro relatório trimestral que enviou de Paris, onde estava a fazer um estágio que o havia de conduzir ao doutorado, dirigido à Junta de Educação Nacional, a entidade que subsidiava a sua estada e a quem tinha de prestar contas periodicamente, caracterizou de forma preocupante o conhecimento recebido por um licenciado em Ciências Matemáticas no Ensino Superior em Portugal:

"ignorância de uma enormidade de conhecimentos basilares; educação enciclopédica, de que resulta o conhecimento superficial de todas as matérias estudadas; ausência quase completa de espírito crítico; ausência de iniciação aos métodos de investigação, de que resulta um interesse nulo pela investigação científica."

Data de aceitação: Setembro de 2021.

Palavras chave. Matemática portuguesa do século XX; António Monteiro; investigação matemática no Brasil e na Argentina no século XX; revistas matemáticas. 
É este grave estado de coisas que Monteiro queria mudar quando completasse a sua formação em Paris (1931-1936) e regressasse a Portugal, o que aconteceu após concluir o seu doutorado sob a orientação do matemático francês Maurice Fréchet $[1]$.

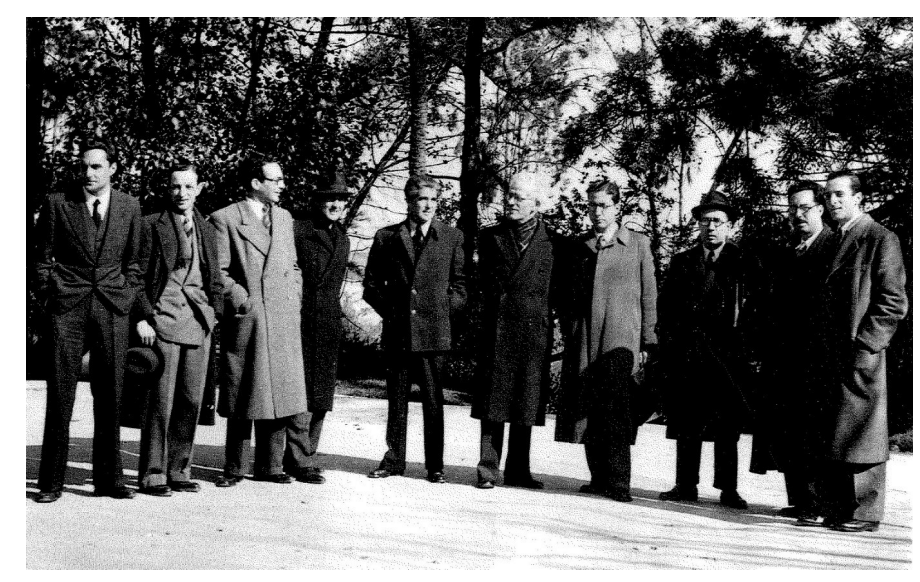

Figura 1. Maurice Fréchet em Portugal em 1942. Foto na Faculdade de Ciências de Lisboa (hoje Museu Nacional de História Natural e da Ciência) com a grande maioria dos matemáticos da chamada geração de 40. Da esquerda para a direita: Hugo Ribeiro, Armando Gibert, António Monteiro, Manuel Zaluar Nunes, Bento de Jesus Caraça, Maurice Fréchet, José Sebastião e Silva, Ruy Luis Gomes, José Ribeiro de Albuquerque e Augusto Sá da Costa. Monteiro foi para o Brasil em 1945, e os restantes, com excepção de Sebastião e Silva e Ribeiro de Albuquerque, seriam excluídos da Universidade na grande purga feita pelo regime em 1946/47.

No regresso a Portugal Monteiro foi o dinamizador principal daquela que ficou conhecida por "Geração de 40" [2], que em dez anos (1936-1945) mudou o panorama matemático português, criando em 1937 um jornal de investigação matemática internacional, a Portugaliae Mathematica, que ainda hoje existe, iniciando um jornal de divulgação matemática em 1940, a Gazeta de Matemática, que igualmente continua a ser publicada [3], e fundando a Sociedade Portuguesa de Matemática (SPM) em 1940. Esse grupo de matemáticos teve uma actividade constante na organização de diversos Seminários sobre temas da actualidade matemática e fundaram diversos centros de pesquisa, criando condições para, de um modo continuado, se poder desenvolver investigação matemática em Portugal sobre temas então actuais [4].

Monteiro considerava que o renascimento da matemática no seu país só seria possível se se criasse uma corrente de fundo entre os estudantes do Ensino Secundário e da Universidade de interesse pela Matemática. Nesse sentido, e igualmente como tentativa de ultrapassar o bloqueamento que era feito na Universidade às propostas de renovação curricular dos matemáticos da nova geração, e seguindo o exemplo dos Estados Unidos para mobilizar a juventude e levá-la a uma prática em que desenvolvesse um espírito crítico virado para a pesquisa, foram criados os Clubes de 
Matemática em 1942. De começo a iniciativa teve muito sucesso, foram fundados clubes em várias universidades e faculdades, mas pouca duração tiveram, pois o Governo, receoso de tudo o que fosse adquirir espírito crítico, fez espalhar o boato que neles só participavam comunistas, o que bastou para em poucos anos fazê-los desaparecer.

A Ditadura queria manter um controle estrito sobre a população, e com a vitória da Frente Popular nas eleições espanholas de Fevereiro de 1936 o regime entrou em pânico, sentindo-se ameaçado por haver em Espanha uma aliança progressista no poder, que incluía os socialistas. Apertou então o seu controle, e fez entrar em vigor um decreto que obrigava todos os que queriam ter emprego no Estado a assinar uma declaração que essencialmente exprimia o apoio ao regime. Monteiro recusou assinar essa declaração, e em consequência ficou impossibilitado de trabalhar para o Estado, nomeadamente não podia ser docente universitário. Deste modo, para ganhar a vida, Monteiro deu explicações particulares. Mas a situação estava muito longe de ser ideal, e Monteiro a partir de certa altura começou a procurar hipóteses de trabalho fora de Portugal, no que foi ajudado por matemáticos estrangeiros.

Acabou por aparecer um convite do Brasil em Setembro de 1943 que ele aceitou, mas as burocracias da ditadura atrasaram cerca de ano e meio a sua partida. Deixou então Portugal, na companhia de sua esposa Lídia, e dos dois filhos, António e Luiz. Teve a cátedra de Análise Superior na Faculdade Nacional de Filosofia da Universidade do Brasil (FNFi), no Rio de Janeiro, futura UFRJ. Nessa altura o seu

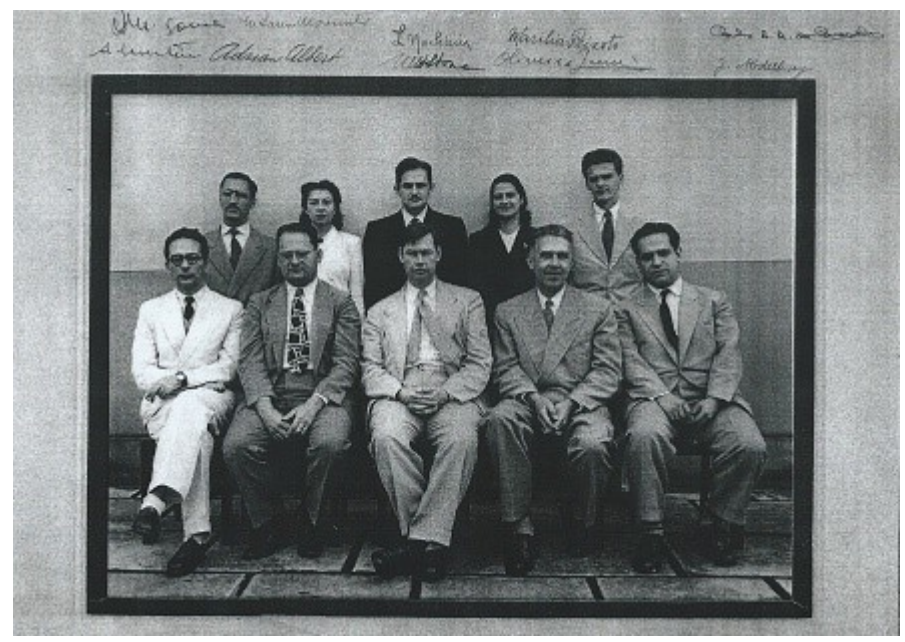

Figura 2. Elementos do Departamento de Matemática da FNFi em 1948. Entre parêntesis está a idade de cada um na altura da foto: De pé: Alvércio Moreira Gomes (32), Maria Laura Mousinho (31), Leopoldo Nachbin (26), Marília Peixoto (27), Carlos Alberto Aragão Carvalho (24). Sentados: António Monteiro (41), Abraham Adrien Albert (43), Marshall Harvey Stone (45), Ernesto Luiz de Oliveira Junior (47), José Abdelhay (31). Foto do espólio de António Monteiro, gentilmente cedida por Jorge Rezende.

Departamento de Matemática era o segundo melhor centro de matemática do Brasil, 
estando o primeiro na Faculdade de Filosofia, Ciências e Letras da Universidade de S. Paulo (FFCL). Assinou um contrato de quatro anos, renovável. O Departamento de Matemática da FNFi teve visitantes estrangeiros importantes, como Abraham Adrien Albert (1905-1972), Professor Catedrático em Chicago e futuro Presidente da American Mathematical Society no biénio 1965/66, e Marshall Harvey Stone (19031989), Professor Catedrático em Harvard, Presidente da American Mathematical Society em 1943/44, e futuro Presidente da International Mathematical Union no período 1952-54. O Presidente do Departamento de Matemática, e responsável pela contratação de Monteiro, bem como da viabilização das visitas de Albert e de Stone, era Ernesto Luiz de Oliveira Junior (1901-?), que tinha sido assistente dos matemáticos italianos Luigi Fantappié (1901-1956) e Giacomo Albanese (1890-1948) na FFCL entre 1934 e 1936. Monteiro deu vários cursos e seminários sobre temas como Topologia Geral, Espaços de Hilbert, Análise Funcional, Álgebras de Boole e Reticulados. Contactou importantes matemáticos, entretanto chegados à FFCL, alguns já conhecidos de Paris, como André Weil (1906-1998) e Jean Dieudonné, (1906-1992) dois elementos fundamentais do grupo Bourbaki.

Monteiro orientou alguns alunos nos anos que esteve no Rio de Janeiro: Maria Laura Mouzinho (mais tarde Maria Laura Leite Lopes) (1917-2013) teve Monteiro por supervisor do seu doutorado, sendo a segunda mulher a obter o doutorado em matemática no Brasil (1949). A primeira tinha sido Marília Peixoto (1921-1961), ao ser aprovada em concurso para livre docente na Escola Nacional de Engenharia [5] em 1948. Além disso teve influência decisiva em Leopoldo Nachbin (1922-1993), contratado em 1947 para docente da FNFi, Maurício Peixoto (1921-2019), da Escola Nacional de Engenharia, o único matemático brasileiro com o qual Monteiro tem um artigo em comum publicado na Portugaliae Mathematica, e Paulo Ribenboim (1928-), também contratado para a FNFi em 1948. Nachbin foi um dos maiores matemáticos brasileiros do século XX, estando na fundação do Centro Brasileiro de Pesquisas Físicas em 1949, na do CNPq e da CAPES em 1951. Foram alunos de Monteiro os dois primeiros matemáticos brasileiros a darem conferências plenárias nos Congressos Internacional de Matemáticos: Leopoldo Nachbin em Estocolmo, 1962, e Maurício Peixoto em Vancouver, em 1974. Os dois, conjuntamente com Lélio Gama, foram co-fundadores do Instituto de Matemática Pura e Aplicada (IMPA) em 1952. Peixoto foi presidente do CNPq, da Academia Brasileira de Ciências e da Sociedade Brasileira de Matemática. Já na Argentina, Monteiro orientou o doutorado de Mário Tourasse Teixeira (1925-1993) que defendeu a sua tese na FFCL da Universidade de S. Paulo em 1965.

Em 1945 e 46 Monteiro foi igualmente investigador no Núcleo Técnico Científico da Fundação Getúlio Vargas, e com Lélio Gama (1892-1981) coordenou o seu Núcleo de Matemática. Participou da fundação da revista de pesquisa matemática Summa Brasiliensis Mathematicae, da qual sairam 2 volumes entre 1946 e 1951: o primeiro diz respeito ao período 1945/46, o segundo é relativo aos anos 1947/51. Estes dois primeiros números têm artigos de Monteiro (com Hugo Ribeiro), Nachbin, Maria Laura, Paulo Ribenboim, Maurício Peixoto e Lélio Gama, mas igualmente de Paul Halmos (1916-2006), Jean Dieudonné (2), Abraham Albert (2), André Weil, Paul Erdös (1913-1996), Jacques Dixmier (1924-) e Oscar Zariski (1899-1986). Depois da 
partida de Monteiro para a Argentina ainda foram publicados mais dois números, o $\mathrm{n}^{0} 3$, com artigos de 1952 a 1956, e o $\mathrm{n}^{0} 4$, relativo ao período de 1957 a 1960 .

Em 1948 iniciou a publicação das Notas de Matemática, sendo o editor dos seis primeiros volumes (1948/49), que incluem dois trabalhos seus, dois de Nachbin, um de Maurício Peixoto e um de José Abdelhay (1917-1996), colega de Monteiro no Departamento de Matemática da FNFi. Do n⿳007, já com Monteiro na Argentina, até ao $\mathrm{n}^{0}$ 47, em 1972, foi Nachbin o editor. A partir daí a North Holland Publishing Company tomou conta das Notas, se bem que Nachbin ainda tenha sido o editor dos primeiros números.

Havia (e continua a haver) no Brasil uma numerosa comunidade portuguesa, e uma parte muito significativa estava declaradamente contra a ditadura em Portugal. Por essa razão o governo português destacou para o Brasil numerosos agentes da sua polícia política para vigiarem os movimentos da comunidade portuguesa e informarem o governo das iniciativas contrárias ao regime que fossem aí organizadas. Monteiro também tomou parte activa em sessões antifascistas. Por isso era pessoa non grata do regime, que procurou dificultar-lhe a vida, fazendo pressão para o seu contrato na Universidade não ser renovado. Outro problema surgiu pelo facto da Universidade, a partir de certa altura, ter começado a pagar-lhe os ordenados com cada vez maior atraso. Monteiro ficou com a sua vida bastante dificultada e começou a estudar as possibilidades de ir trabalhar para outro país. No fim de 1948, por meio do matemático espanhol Pedro Pi Calleja (1907-1986) [6], chegou-lhe uma oferta de emprego na Universidade de Cuyo, em S. Juan, na Argentina.

Em 1949 tornou-se investigador do Centro Brasileiro de Pesquisas Físicas (CBPF) que passou a distribuir o Summa Brasiliensis Mathematicae. O CBPF era uma alternativa ao ambiente conservador da FNFi. A pressão feita sobre a Universidade acabou por ter efeito, e o contrato de Monteiro, depois de vários atrasos, não foi renovado, o que o deixou sem meios de subsistência. Para ter um ordenado regular, passou a trabalhar na Companhia de Aviação Transcontinental, onde fez cálculos estatísticos, utilizou a programação linear para optimizar a procura de lugares, e elaborou roteiros de voo. Mas era claramente uma solução provisória, até ser possível a sua saída para uma universidade noutro país.

Finalmente parte para a Argentina, onde chega a 5 de Dezembro. Aqui vai ter uma acção importante na modernização da matemática deste país. Ficou em S. Juan de 1950 a 1956, leccionando na Universidade de Cuyo, tendo igualmente dinamizado a criação em 1951 de um Departamento de Investigações Científicas, sediado em Mendoza, que incluía um Instituto de Matemática. Em 1955 colaborou na criação da Revista Matemática Cuyana. A partir de 1957 mudou-se para Bahia Blanca, contratado pela Universidad del Sur (UNS). Organizou a licenciatura em Matemática e o Instituto de Matemática, estruturou e organizou a sua biblioteca, que passou a ser a mais completa da América do Sul, e criou duas séries de publicações da UNS, que incluiam preprints e apontamentos de seminários avançados de pesquisa: Notas de Lógica Matemática em 1964, e Notas de Álgebra e Análise em 1966. Estabeleceu um regime de permutas com outras instituições. A qualidade e o quantidade dos artigos incluídos nos números destas duas publicações eram tais que se conseguiram desse modo alguns dos mais caros e reputados jornais estrangeiros. Em 1970, das 453 
publicações que a Biblioteca do Instituto de Matemática recebia, cerca de metade era obtida por troca com publicações criadas pelo matemático português.

Monteiro tinha dito que nunca regressaria a Portugal enquanto ele estivesse sob o domínio da ditadura. Esta foi derrubada na revolução de 1974, pelo que a partir daí passou a ser viável a Monteiro visitar o seu país. Foi o que fez em 1977, ficando em Portugal dois anos como investigador do Centro de Matemática e Aplicações Fundamentais. Escreveu um artigo, "Sur les Algèbres de Heyting Symmétriques", que obteve em 1978 o Prémio Gulbenkian de Ciência e Tecnologia. Regressou em 1979 à Argentina, e faleceu pouco depois, a 29 de Outubro de 1980, em Bahia Blanca.

Em reconhecimento do muito que fez pela matemática argentina e pela sua modernização, e em particular pela sua universidade, a UNS deu o seu nome à Biblioteca do Instituto de Matemática. Desde 1991 que a UNS organiza, de dois em dois anos, um congresso com o nome Dr António Monteiro. Em cada Encontro é colocado ênfase num ramo diferente da matemática, para assim se ir percorrendo a matemática na sua variedade.

Em 2006, na sessão Ibero-American mathematics in the 19th and 20th centuries, no Congresso Internacional dos Matemáticos de Madrid, foi feita uma homenagem a Monteiro, e simultaneamente foram lançadas as suas obras em livro (número limitado de exemplares) e em CD-ROM [7], numa edição conjunta da Fundação Calouste Gulbenkian e da Humboldt Press de Londres. O CD ROM só ficou comercialmente disponível em 2008.

Podemos terminar com duas citações, uma de um seu aluno argentino, outra de um aluno brasileiro. Ambas dão conta não só da grande influência matemática de Monteiro, respectivamente na Argentina e no Brasil, mas igualmente revelam o carisma deste matemático que se dedicava por completo à missão que se tinha atribuído, e que, pelo seu empenho e atitude, modificava a vida daqueles que com ele trabalhavam.

Roberto Cignoli (1938-2018), um dos alunos argentinos de Monteiro, escreveu no prefácio da excelente Fotobiografia que a Sociedade Portuguesa de Matemática publicou em 2007 [8], na celebração dos cem anos do nascimento de António Monteiro:

"António Aniceto Monteiro dejó profundas huellas de su paso por los tres países en los que actuó: Portugal, Brasil y Argentina. En los tres debió afrontar penurias morales y económicas, que no consiguieron doblegar su espiritú de luchador incansable en pro de la investigación matemática, la que consideraba un aporte esencial para el desarollo científico de los países."

Igualmente é muito significativo o depoimento prestado por Paulo Ribenboim nos Anais do Encontro Internacional realizado em 2007 em Lisboa para celebrar os cem anos do nascimento de António Monteiro e publicados como número especial do Boletim da SPM [9]. Por questões de saúde não foi possível a Ribenboim viajar para participar no Encontro, mas não quis deixar de marcar presença e enviou um texto para ser lido no seu início, intitulado "Lembrando Monteiro", que conclui deste modo:

"Aos jovens, o carisma e a honestidade intelectual de Monteiro eram exemplares e um modelo que não me abandonou.[...] O Monteiro foi o primeiro - como (não) se diz em inglês "the first but not the least" - de uma trindade 


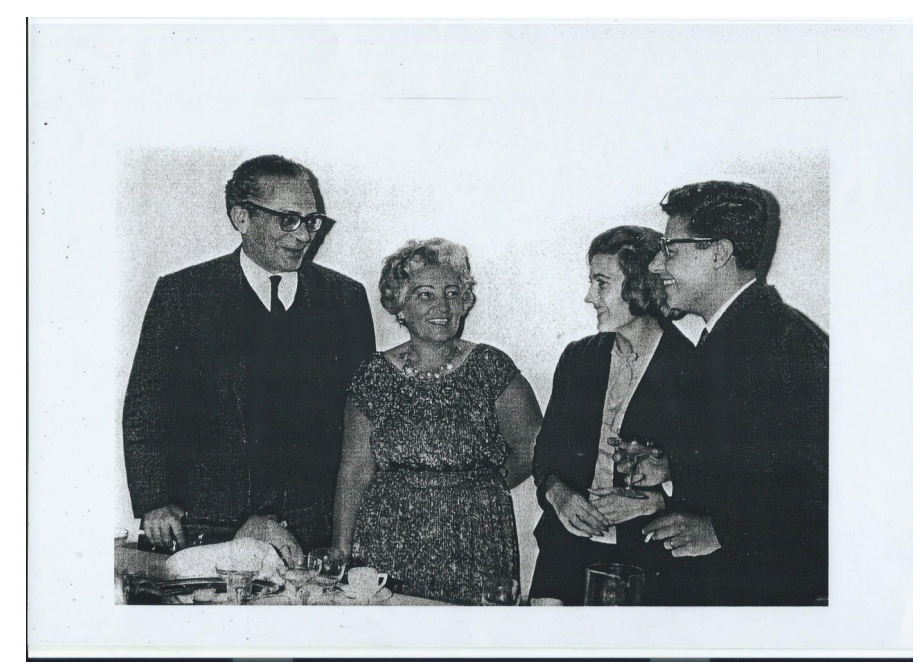

Figura 3. António Monteiro (à esquerda) e sua esposa, Lídia Monteiro, no casamento de seu aluno Roberto Cignoli (à direita), aqui com a esposa, Carmen Cignoli. Foto de Maio de 1965. Foto do espólio de António Monteiro, gentilmente cedida por Jorge Rezende.

de matemáticos que me formaram, juntamente com o Dieudonné e o Krull, a quem devo uma grande parte do que me tornei. Minha opinião sobre os "grandes homens" é clara: não são os generais, nem os politicos, mas os cientistas, artistas e os matemáticos, que são os cientistas-artistas. Monteiro foi um grande homem."

António Aniceto Monteiro foi de facto uma figura notável da história científica do século XX, deixando profundamente gravada a sua acção em Portugal, no Brasil e na Argentina.

Notas

[1] A sua tese intitula-se "Sur l'aditivité des noyaux de Fredholm".

[2] Nesta geração pontificaram, entre outros, Hugo Ribeiro (1910-1988), Ruy Luis Gomes (1905-1984), Bento de Jesus Caraça (1901-1948), Armando Gibert (19141985), Manuel Zaluar Nunes (1907-1967), João Remy Freire (1917-1992), José Sebastião e Silva (1914-1972) e José da Silva Paulo (1905-1976).

[3] Embora a publicação não tenha sido continua.

[4] "Núcleo de Matemática, Física e Química" (1936), "Centro de Estudos Matemáticos Aplicados à Economia" (1938), "Seminário de Análise Geral" (1939), "Centro de Estudos Matemáticos de Lisboa" (1940), "Centro de Estudos Matemáticos do Porto" (1942). Igualmente começaram a ser publicados em 1943 os Cadernos de Análise Geral com trabalhos deste grupo de matemáticos em cinco séries: 'Álgebra Moderna, Topologia Geral, Teoria da Medida e Integração, Geometria das Distâncias, e Teoria das Estruturas e Problemas de Fundamentos.

[5] Na época o grau de livre-docente era equivalente ao de doutor. Agradeço esta informação ao Professor Clóvis Pereira da Silva.

[6] Matemático espanhol que abandonou a Espanha no fim da Guerra Civil e emigrou, primeiro para França, onde trabalhou no Instituto Henri Poincaré, e depois, 
devido ao começo da $2^{\text {a }}$ Guerra Mundial, para a América do Sul. Esteve primeiro em Cuba e depois fixou-se na Argentina em 1942, onde permaneceu até 1956, tendo então regressado a Espanha.

[7] The Works of António A. Monteiro (editores: E. Ortiz, A. Pereira Gomes e J P Kahane), Fundação Calouste Gulbenkian, Lisbon- The Humboldt Press, London, 2008.

[8] António Aniceto Monteiro Uma fotobiografia a várias vozes/una fotobiografía a varias voces (coordenadores: J. Rezende, L. Monteiro e E. Amaral), Sociedade Portuguesa de Matemática, Lisboa, 2007. Jorge Rezende tem neste momento um artigo muito desenvolvido sobre a passagem de Monteiro no Brasil, intitulado "O Estrangeiro Indesejável: O Brasil e António Aniceto Monteiro (lutas, tramas, diplomacias e polícias)" a sair brevemente no número 39 de Cultura, Revista de História e Teoria de Ideias, publicação do CHAM, Centro de Humanidades.

[9] Actas do Colóquio do Centenário de António Aniceto Monteiro (1907-1980), Editor: Luis Saraiva, Boletim da Sociedade Portuguesa de Matemática, Número Especial, Lisboa, 2008. Estes Anais incluiem o artigo de António Videira "António Monteiro no Brasil (1945-1949): uma breve passagem com resultados duradouros", pp. 183-211. O número 19 da Portugaliae Mathematica, de 1980, de homenagem a Monteiro, inclui o artigo de Leopoldo Nachbin "The Influence of António A. Ribeiro Monteiro in the Development of Mathematics in Brazil", pp. XV-XVII.

Luís Saraiva é Professor Associado do Departamento de Matemática da Faculdade de Ciências da Universidade de Lisboa e investigador do Centro Interuniversitário de História da Ciência e da Tecnologia (CIUHCT).

Email address: lmsaraiva@fc.ul.pt 